\title{
Noma disease: 10 years of research in the quest of a microbial etiology
}

\author{
P François ${ }^{1^{*}}$, K Whiteson $^{2}$, D Baratti Mayer ${ }^{3}$, J Schrenzel ${ }^{4}$, D Pittet ${ }^{5}$, the GESNOMA study group \\ From 3rd International Conference on Prevention and Infection Control (ICPIC 2015) \\ Geneva, Switzerland. 16-19 June 2015
}

\section{Introduction}

Noma is a devastating ancient illness that causes severe facial disfigurement in $>140,000$ children every year mainly in Africa, South America and India. The cause of noma remains unknown but infection, oral hygiene and immune status likely all contribute

\section{Objectives}

Efforts have been deployed over the 2 past decades to identify microbial agents (fungal, bacterial and viral) responsible for noma

\section{Methods}

Research in the field of microbial identification has benefited from dramatic technical improvements. Until the late 90's culture based methods were predominantly used. More recently, culture-independent methods were used to identify bacteria in noma patients. We present three approaches used to study hundreds of gingival samples from noma patients and local control samples collected from villages near Zinder, Niger (Africa): large-scale cloning sequencing methods used at the beginning of 2000, high-density microarrays and high-throughput sequencing (HTS) devices

\section{Results}

Culture-based methods identified Fusobacterium necrophorum as the causative agent of noma, while cultureindependent methods identify higher levels of Fusobacteriales in healthy controls. Cloning-sequencing strategies identify disequilibrium in microbial communities from noma patients particularly in Fusobacteria, Prevotella intermedia and Peptostreptococcus genus abundance. This was also detected in studies using semiquantitative microarrays. More recently, the utilization of
HTS provided a detailed analysis of microbial flora in noma patients and identified Clostridiales, Bacteroidales, and Spirochaetales as indicators of noma. Our epidemiological investigations excluded the possible role of viruses such as cytomegalovirus or morbillivirus as possible significant contributor of noma disease

\section{Conclusion}

Although we identify a unique distribution and composition of microbial communities in noma wound sites compared to unaffected samples from the same mouths and healthy controls, the etiology of noma disease remains elusive. Future studies should include longitudinal sampling in high risk areas and detailed exploration of microbiota before and during development of lesions

\section{Disclosure of interest}

None declared.

\section{Authors' details}

${ }^{1}$ Medical Specialties, Univ Hosp of Geneva, Geneva, Switzerland. ${ }^{2} \mathrm{Mol}$ Biol \& Biochem, Unive of California, Irvine, USA. ${ }^{3}$ Plastic Surgery, Univ Hosp of Geneva, Geneva, Switzerland. ${ }^{4}$ Dep. Medical Specialties, Univ Hosp of Geneva, Geneva, Switzerland. ${ }^{5}$ Cont \& Prev Infection, Univ Hosp of Geneva, Geneva, Switzerland.

Published: 16 June 2015

doi:10.1186/2047-2994-4-S1-P258

Cite this article as: François et al.: Noma disease: 10 years of research in the quest of a microbial etiology. Antimicrobial Resistance and Infection Control 2015 4(Suppl 1):P258. 